\title{
Accurate Transmission Loss Allocation Algorithm Based on the Virtual Transaction Strategy: Comparison of Path-integral with Discrete Integral Methods
}

\author{
Kyung-Il Min* and Young-Hyun Moon ${ }^{\dagger}$
}

\begin{abstract}
This paper presents a new algorithm to determine accurate bus-wise transmission loss allocation utilizing path-integrals dictated by the transaction strategy. For any transaction strategy, the total sum of the allocated transmission losses of all buses is equal to the actual loss given by the AC powerflow calculation considering the distributed slack. In this paper, the bus-wise allocation of the transmission loss is calculated by integrating the differential loss along a path determined by the transaction strategy. The proposed algorithm is also compared with Galiana's method, which is the well-known transmission loss allocation algorithm based on integration. The performance of the proposed algorithm is evaluated by case studies carried out on the WSCC 9-bus, IEEE 14-bus, New England 39-bus, and IEEE 118-bus systems. The simulation results show that the proposed algorithm is fast and accurate with a large step size.
\end{abstract}

Keywords: Transmission loss allocation, Average loss sensitivity factor, Marginal loss sensitivity factor, Path dependency, Path independency, Transaction strategy

\section{Introduction}

Since the 1990s, competitive market systems of power transactions have been introduced to improve the efficiency of power industries over the world. The efficiency of the power market system is based on reasonable pricing that guarantees fair competition between the suppliers and the consumers in power transactions. As most power transactions are conducted by spot trading, that there should be reasonable spot pricing to encourage market participants to react based on real-time situations is very important. Locational marginal pricing (LMP) is one of the relevant issues related to this topic in power market systems [1]-[3]. About $3 \%-5 \%$ of the total power generated is lost in transmission, making transmission loss one of the major factors in LMP. A factor of LMP is assigned in proportion to each allocated loss, which may considerably affect the competitive position of the Gencos. Nevertheless, the spot price hardly ever reflects transmission loss because the allocation of transmission loss is a nonlinear and path-dependent problem with non-unique solutions. Most of the proposed conventional theories based on sensitivity analysis cannot deal with the nonlinear aspect of the problem, and the sum of the allocated losses results in twice the loss [4]-[10]. Recently, many studies have been conducted based on various approaches such as circuit theory [11]-[13], game theory [14], integration method [15]-[17], etc [18], [19].

\footnotetext{
$\dagger$ Corresponding Author: Department of Electrical and Electronic Engineering, Yonsei University, Korea. (moon@yonsei.ac.kr)

* Facility Planning Team, Semiconductor, Samsung Electronics Inc., Korea. (kimin@yonsei.ac.kr)

Received: February 5, 2010; Accepted: September 30, 2010
}

This paper shows that the total transmission loss can be represented by the sum of the bus-wise partial integration calculated by real power injections. Each of these partial integrations is equal to the integration of the incremental loss with respect to the sensitivity coefficient, considering the nonlinearity.

The idea of determining loss allocation by integration can be found in the literature [15], [20]-[21]. Galiana [20], [21] introduced power transactions to calculate loss allocation by integration and discussed why path dependency should be considered. However, his method seems to fail to reduce sufficiently the discrepancy between the sum of the allocated losses and the system loss without any normalization. This paper details the differences between the proposed method and that of Galiana. Cheung [15] attempted to reduce the difference between the total allocated loss and the system loss by integrating Newton's penalty factor. However, the integration equations in his paper did not consider path dependency.

This paper proposes a line integral method that considers the path dependency of transmission loss allocation. The path dependency dictates that allocated losses vary with integral paths. These variations in the allocated losses may cause a problem in power market systems, making it more difficult to establish the relevant theory. The main factor in determining the integral paths is the bilateral power transaction strategy. This paper also reveals that the execution time for the proposed method can be reduced by curtailing the integral step size.

In this paper, the slack-bus-independent loss sensitivity factor [22], [23] is adopted to minimize the variation of the sensitivity with the selection of the slack bus. Instead of a 
transaction matrix, this paper employs a transaction ratio matrix that provides information on the ratio of the generation (the load) supplying for (receiving from) each load (generator). Note that previously settled transactions cannot be applied to the transaction variation in practical systems because loss is allocated after the transaction [14]. Thus, the use of a "ratio" can provide more flexibility when considering transaction variations. This paper introduces a new concept of virtual transactions to achieve more reasonable loss allocations than the actual bilateral contracts, which can be well applied for incomplete bilateral markets including pool markets.

Throughout this study, AC power flow analysis is used with full consideration of the reactive powers to achieve high accuracy in calculations. The distributed slack bus [22], [23] is employed to remove the slack-bus dependency in loss allocation.

The proposed algorithm is tested for various sample systems including the WSCC 9-bus, IEEE 14-bus, New England 39-bus, and IEEE 118-bus systems. The test results show that considering the nonlinearity of the loss function to cover up the weakness of sensitivity analysis in conventional algorithms is possible.

\section{Conventional Incremental Transmission Loss Method: Use of Discrete Integral}

Incremental transmission loss (ITL) has some advantages that consider network topology and transactions in transmission loss allocation. Many studies on ITL have been conducted [15], [20]-[21]. Particularly, Galiana's research [20] is reviewed and analyzed in this section.

Incremental contractual loss is given as follows:

$$
d L_{i j / k}=\frac{\left(\alpha_{j}-\alpha_{i}\right)}{\alpha_{k}} d G D_{i j}
$$

where $G D$ is the contract matrix, and $\alpha$ is the null space vector.

Using $G D$, the vector or bus load, $\mathbf{P}_{\mathbf{L}}$, can be represented as

$$
\mathbf{P}_{\mathbf{L}}=\mathbf{G D}^{T} \mathbf{1}
$$

The generation vector can also be expressed as follows:

$$
\mathbf{P}_{\mathbf{G}}=\mathbf{G D} \cdot \mathbf{1}+\mathbf{P}_{\mathbf{G}}^{\text {Loss }}
$$

When $k$ is slack, $\alpha$ is defined as

$$
\alpha_{i}=1-\left[\frac{\partial P_{\text {loss }}}{\partial P_{i}}\right]_{k}
$$

The loss of contract $i-j$, whose loss is supplied by bus $k$, can be obtained by integrating (1) for $0 \leq t \leq t_{f}$.

$$
L_{i j / k}=\int_{t=0}^{t_{f}}\left[\frac{\left(\alpha_{j}-\alpha_{i}\right)}{\alpha_{k}}\right] \frac{d G D_{i j}}{d t} d t
$$

Remarks: Galiana expressed his algorithm as a time integral, although it is actually a discrete integral. An acceptance order of the transaction is used and integrated with respect to every transaction change, as seen in Fig. 1.

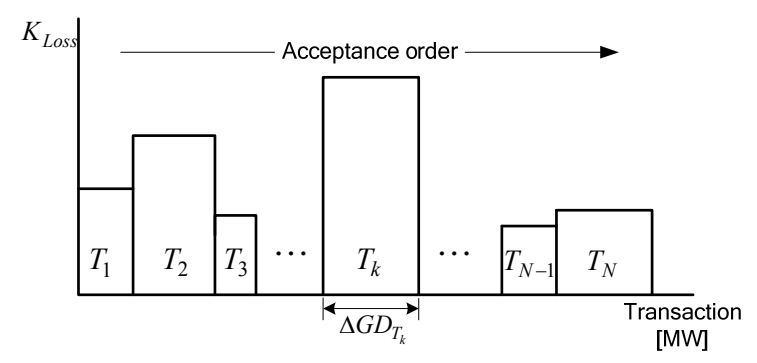

(a) Discrete integral

\begin{tabular}{c|c}
\hline $\begin{array}{c}\text { Acceptance } \\
\text { Order }\end{array}$ & Transaction \\
\hline $\mathrm{T}_{1}$ & $\mathrm{GD}_{12}$ \\
$\mathrm{~T}_{2}$ & $\mathrm{GD}_{14}$ \\
$\vdots$ & $\vdots$ \\
$\mathrm{T}_{\mathrm{N}}$ & $\mathrm{GD}_{69}$ \\
\hline Sum & $\mathrm{P}_{\mathrm{D}}$ \\
\hline
\end{tabular}

(b) Acceptance order

Fig. 1. Illustrative example of the discrete integral method.

The above discrete integral in (5) can be expressed in the form of summation with order

$$
L_{i j / k}=K_{\text {Loss }}^{(k)}(\mathbf{P}, \mathbf{Q}) \Delta G D_{i j}^{(k)}
$$

where $K_{\text {Loss }}=\left(\alpha_{j}-\alpha_{i}\right) / \alpha_{k}$ evaluated at bus injections $\mathrm{P}$, and $\mathrm{Q}$ determined by the accepted transactions at the $k$-th stage.

In the above equation, the loss sensitivity $K_{\text {Loss }}^{(k)}$ should be computed with the bus injections composed by the transactions already accepted in the $k$-th step. The total loss can be calculated by accumulating all the areas from $T_{1}$ to $T_{\mathrm{N}}$, represented as follows:

$$
P_{\text {Loss }, t t l}=\sum_{k=1}^{N} K_{\text {Loss }}^{(k)} \cdot \Delta G D_{i j}^{(k)}
$$

where $N$ is the number of transaction.

The integration step of discrete integral should be decreased to achieve high accuracy in calculation. However, this results in a drastic increase in the computational burden. Consequently, Galiana's method can hardly be applied 
to large power systems.

Galiana's method can be performed using the procedure shown in Fig. 2.

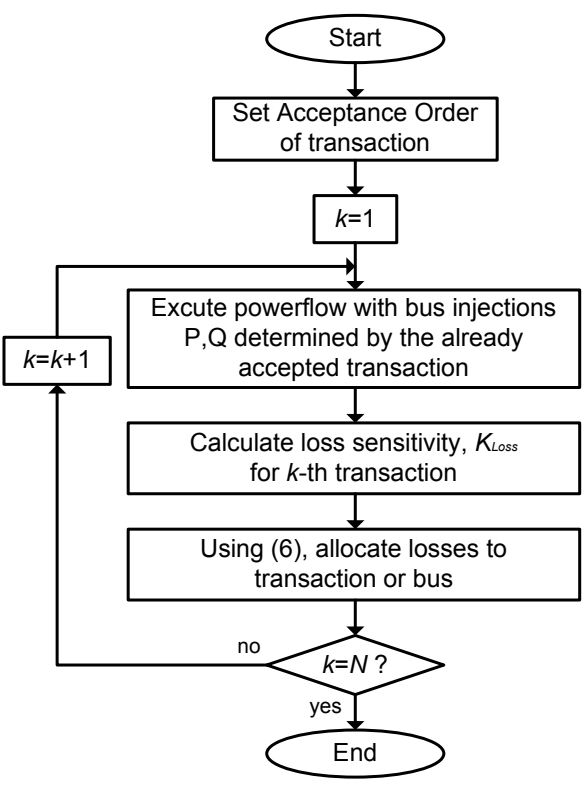

Fig. 2. Loss allocation procedure of Galiana's method.

\section{Theory of Loss Allocation Considering Path Dependency: Use of Path Integral}

Total transmission loss in power systems can be uniquely determined by $\mathrm{AC}$ power-flow techniques with the current system states and has constant values regardless of historical paths, such as load increases and generation distributions, indicating that the total system loss is path independent. However, the nonlinearity of line losses causes bus-wise allocated losses to vary considerably as to "which generation or load increased ahead," i.e., the buswise allocated transmission losses are path dependent. These two contrary results make it confusing to develop an exact theory that provides accurate bus-wise allocated losses, of which the sum is only equal to the total transmission loss [4]-[10], [15], [20]-[21], [24]-[27]. This section presents the proposed mathematical theory satisfying both the path independency of the total transmission loss and the path dependency of bus-wise allocated losses.

\subsection{Mathematical Approach to Path Dependency}

The general path dependency in mathematics with a scalar function $f\left(x_{1}, x_{2}, \ldots, x_{\mathrm{n}}\right)$ can be expressed as follows:

$$
P=f\left(x_{1}, x_{2}, \cdots, x_{n}\right)
$$

with $f(0,0, \ldots, 0)=0$.

The total differential of $P$ is given by

$$
d P=\frac{\partial f}{\partial x_{1}} d x_{1}+\frac{\partial f}{\partial x_{2}} d x_{2}+\cdots+\frac{\partial f}{\partial x_{n}} d x_{n}
$$

$P$ can be rebuilt by integrating (9), as shown below:

$$
P=\int_{c}^{\mathbf{x}} d P=\int_{c} \int_{(0, \cdots, 0)}^{\left(x_{1}, \cdots, x_{n}\right)}\left(\frac{\partial f}{\partial x_{1}} d x_{1}+\frac{\partial f}{\partial x_{2}} d x_{2}+\cdots+\frac{\partial f}{\partial x_{n}} d x_{n}\right)(10)
$$

Here, $P$ is uniquely determined by state $\mathbf{x} \in \mathbb{R}^{n}$, and it becomes path independent even if it is represented by an integral form. Eq. (10) is a line integral, where $c$ is an integral path (contour). The condition of path independency is given by Clairaut's theorem [28] as follows:

$$
\frac{\partial}{\partial x_{i}}\left(\frac{\partial f}{\partial x_{j}}\right)=\frac{\partial}{\partial x_{j}}\left(\frac{\partial f}{\partial x_{i}}\right), \text { for all } i, j
$$

As $f(\mathbf{x})$ in (8) is a well-defined function uniquely determined by state $\mathbf{x}$, it satisfies (9) for all $i, j$. Therefore, (10) is path independent.

However, one term of (10) can be expressed as follows:

$$
P^{(i)}=\int_{c}^{\mathbf{x}} \frac{\partial f(\mathbf{x})}{\partial x_{i}} d x_{i}
$$

In (12), a line integral for $x_{i}$ should be evaluated along path $c$ because $\partial f / \partial x_{i}$ is a function of $\mathbf{x}$. Therefore, each term of the line integral in $(10), P^{(i)}$, is dependent on path $c$, whereas $P$ itself in (10) is path independent.

\subsection{System Loss and Bus-wise Losses}

The total transmission loss in a power system can be calculated by AC power-flow techniques with bus injection power represented as follows:

$$
P_{l o s s, t t l}=P_{l o s s}\left(\theta_{2}, \cdots, \theta_{n}, V_{G 1}^{s}, \cdots, V_{G m}^{s}, V_{m+1}, \cdots, V_{n}\right)
$$

Here, it is assumed that Bus 1 is a slack and Buses 2 to $m$ are voltage-controlled buses. The specified variables $V_{G 1}^{s}, \cdots, V_{G m}^{\varsigma}$, and $\theta_{1}$ can be exempted from the independent variable because they are specified as constants.

The system loss can be regarded as a function of bus injections because bus injections completely determine the system state in (13).

$$
P_{\text {loss }, t l l}=P_{\text {loss }}\left(P_{2}, \cdots, P_{n}, Q_{m+1}, \cdots, Q_{n}\right)
$$

Note that the function $P_{\text {loss }}$ in (14) is hardly obtained in a closed form, but partial differential terms of (14) can be calculated easily. However, $P_{\text {loss }}$ is uniquely determined by bus injections, and it becomes a well-defined function. That $P_{\text {loss }}$ is hardly obtained as a closed form is another matter. 
In the same manner as in (10), $P_{\text {loss,ttl }}$ in (14) can be rewritten by the sum of the partial integrals:

$$
\begin{aligned}
P_{\text {loss }, t t l} & =\int_{c} \int_{(0, \cdots, 0,0, \cdots, 0)}^{\left(P_{2}, \cdots, P_{n}, Q_{m+1}, \cdots, Q_{n}\right)} d P_{\text {loss }} \\
= & \int_{c}^{(\mathbf{P}, \mathbf{Q})}\left(\sum_{i=2}^{n} \frac{\partial P_{\text {loss }}}{\partial P_{i}} d P_{i}+\sum_{i=m+1}^{n} \frac{\partial P_{\text {loss }}}{\partial Q_{i}} d Q_{i}\right)
\end{aligned}
$$

where $\mathbf{P}=\left[P_{2}, \cdots, P_{n}\right]^{T}, \mathbf{Q}=\left[Q_{m+1}, \cdots, Q_{n}\right]^{T}$.

In (15), the total sum of the partial integral terms is path independent, but each partial integral term is path dependent for path $c$. Here, the loss sensitivity factors for $\mathbf{P}$ and $\mathbf{Q}$ are defined as follows:

$$
\begin{aligned}
& K_{P_{i}}^{\text {loss }}=\frac{\partial P_{\text {loss }}}{\partial P_{i}} \\
& K_{Q_{i}}^{\text {loss }}=\frac{\partial P_{\text {loss }}}{\partial Q_{i}}
\end{aligned}
$$

The allocated loss to the $i$-th bus is given as

$$
P_{\text {loss }(i)}=\int_{c}^{(\mathbf{P}, \mathbf{Q})} d P_{(\text {loss }(i)}=\int_{c}^{(\mathbf{P})} \int_{(\mathbf{0}, \mathbf{0})}^{(\mathbf{Q})}\left(K_{P_{i}}^{\text {loss }} d P_{i}+K_{Q_{i}}^{\text {loss }} d Q_{i}\right)
$$

$P_{\text {loss }(i)}$ is path dependent corresponding to (12). The total transmission loss is path independent from (15), and it must equal the sum of the allocated losses.

\subsection{Loss Sensitivity Factor}

In the previous section, the bus-wise allocation of transaction losses was expressed by integrating loss sensitivity factors. This method also requires a specified slack bus. If another slack bus is selected, the loss sensitivity factors may vary significantly. In addition, the reactive powers of the load buses are determined by the load power factors. Thus, the independent variables for system losses become active injection powers only. Previous research on the calculation of the slack bus-independent loss sensitivity factor can be found in the literature [22], [23]. Based on these studies, the loss sensitivity factors for an $i$-th generator bus can be obtained as follows:

$$
\begin{aligned}
S_{p G i}(\mathbf{P}) & =\frac{\partial P_{\text {loss }}(\boldsymbol{\theta}, \mathbf{V})}{\partial P_{G i}}=\left[\left(\frac{\partial P_{\text {slack }}(\boldsymbol{\theta}, \mathbf{V})}{\partial \boldsymbol{\theta}}\right)^{T},\left(\frac{\partial P_{\text {slack }}(\boldsymbol{\theta}, \mathbf{V})}{\partial \mathbf{V}}\right)^{T}\right]\left[\begin{array}{c}
\frac{\partial \boldsymbol{\theta}}{\partial P_{G i}} \\
\frac{\partial \mathbf{V}}{\partial P_{G i}}
\end{array}\right] \\
& =\left[\left(\frac{\partial P_{\text {slack }}(\boldsymbol{\theta}, \mathbf{V})}{\partial \boldsymbol{\theta}}\right)^{T},\left(\frac{\partial P_{\text {slack }}(\boldsymbol{\theta}, \mathbf{V})}{\partial \mathbf{V}}\right)^{T}\right] J^{-1}(\boldsymbol{\theta}, \mathbf{V})\left[\begin{array}{c}
\mathbf{u}_{i}-\boldsymbol{\alpha}_{\mathbf{L}}^{(i)} \\
-\boldsymbol{\beta}_{\mathbf{L}}^{(i)}
\end{array}\right]
\end{aligned}
$$

where

$J(\boldsymbol{\theta}, \mathbf{V})$ Jacobian matrix in the power-flow calculation $\mathbf{u}_{i}$ unit vector, of which the $i$-th element is equal to 1 , i.e., $\mathbf{u}_{i}=\left[\begin{array}{lllllll}0 & \cdots & 0 & 1 & 0 & \cdots & 0\end{array}\right]^{T}$

$\boldsymbol{\alpha}_{\mathbf{L}}^{(i)}$ Load distribution vector corresponding to $\Delta P_{G i}$, i.e., $\alpha_{L k}^{(i)}=\Delta P_{L k}^{(i)} / \Delta P_{G i}=P_{L k} / \sum P_{L k}$.

$\boldsymbol{\beta}_{\mathbf{L}}^{(i)}$ Reactive load distribution vector.

The derivation of (19) is given in references [22], [23]. Although active injection power $\left(P_{i}\right)$ is an independent variable, it is not explicitly included in (19). However, once all injection powers $\left(P_{i} \mathrm{~s}\right)$ are determined, the loss sensitivity factor can be calculated uniquely. The following calculation procedures are implemented to determine the loss sensitivity factor:

Step 1: Once active powers of generation and load for all buses, reactive powers of all loads can be calculated by load power factors. Accordingly, all the injection powers for AC power-flow are provided.

Step 2: Using $\mathbf{P}$ and $\mathbf{Q}$ determined in step 1, the AC powerflow is conducted to determine the state variables $\boldsymbol{\theta}$ and $\mathbf{V}$.

Step 3: Determine $\boldsymbol{\alpha}_{\mathbf{L}}^{(i)}$ for all the buses. If there is a transaction strategy, $\boldsymbol{\alpha}_{\mathbf{L}}^{(i)}$ can be easily determined (as discussed later); otherwise, $\boldsymbol{\alpha}_{\mathbf{L}}^{(i)}$ is determined to distribute the generation increase $\Delta P_{G i}$ proportionally to all the loads.

Step 4: The differential increase of the reactive loads is determined using $\boldsymbol{\alpha}_{\mathbf{L}}^{(i)}$ and $\boldsymbol{\beta}_{\mathbf{L}}^{(i)}$ corresponding to $\Delta P_{G i}$.

Step 5: Loss sensitivity factors are calculated using $\boldsymbol{\theta}$ and $\mathbf{V}$.

Here, the loss sensitivity for the load buses, $\mathrm{S}_{p L i}(\mathbf{P})$, can be similarly calculated. As mentioned above, the loss sensitivity factor can be calculated from only the active power injections, and the bus-wise loss can be determined by the loss sensitivity factors. The allocated losses to a generator bus can be obtained by the following:

$$
P_{\text {loss }(i)}=\int_{c}^{\left(P_{1}, \cdots, P_{n}\right)} S_{p G i}(\mathbf{P}) d P_{G i}
$$

Note that bus-wise losses are dependent on path $c$. The integral path is determined using the historical changes of $P_{i}$, as well as the transaction strategy. Assuming that all of the generations and the loads increase proportionally, the problem can be simplified, and the transaction strategies can be used to determine the path. Using path parameter $\lambda$, a point on the straight path $\bar{c}$ is given as follows:

$$
\bar{P}_{G i}=P_{G i} \lambda
$$

Here, the path parameter $\lambda$ is the same as the loading factor. Given a loading factor $\lambda$, the marginal loss sensitivity is calculated by (19). The marginal loss sensitivity then becomes a function of $\lambda$.

The average loss sensitivity is defined as the mean of the marginal loss sensitivity varying with the loading condition. That is, 


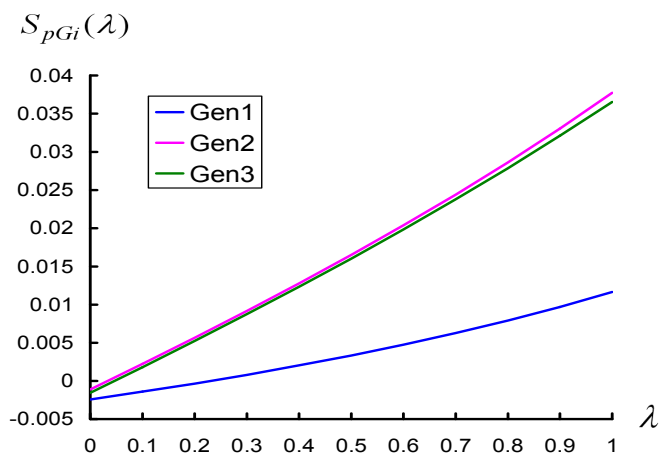

Fig. 3. Trend of loss sensitivity factors for the WSCC 9bus system (case 1).

$$
\bar{S}_{p G i}=\int_{0}^{1} S_{p G i}(\lambda) d \lambda
$$

The trapezoidal method or the Simpson method can be used for the integration to calculate the average loss sensitivity. For $\lambda$ in the range of $[0,1]$, marginal loss sensitivities should be repeatedly calculated at an appropriate integration interval. The loss sensitivity factor increases with $\lambda$ almost linearly, as illustrated in Fig. 3. Therefore, the accuracy of the integration is very insensitive to the integration interval, which allows us to take a relatively large interval, e.g., $\Delta \lambda=0.2$. For large power systems, the computation time can be reduced by adjusting the integration interval.

Bus-wise losses are obtained using the following equation:

$$
P_{\text {loss }(i)}=\bar{S}_{p G i} P_{G i}
$$

\section{Remarks: Compensation for zero-loading loss}

If the system loss at zero loading is zero, no loss compensation is required. However, even at the zero-loading condition, line charging currents always incur some amount of power loss, requiring a corresponding compensation in the loss allocations. This paper adopts a method to distribute the zero-loading loss to each generator in proportion to the dispatched generation because the zero-loading loss is relatively small. Bus-wise loss allocations are modified as follows:

$$
P_{\text {loss }(i)}^{\text {Mod }}=P_{\text {loss }(i)}+\frac{P_{G i}}{\sum P_{G k}} P_{\text {loss }}^{\text {NoLoad }}=\bar{S}_{p G i} P_{G i}+\alpha_{G i}^{(i)} P_{\text {loss }}^{\text {NoLoad }}
$$

where $P_{\text {loss }}^{\text {No Load }}$ is the system loss at the zero-loading condition.

\section{Loss Allocation Considering Transaction Strategy}

\subsection{Transaction Strategy for Loss Allocation}

The transaction strategy [16] for bilateral transactions should be considered to achieve more reasonable transmis- sion loss allocation. In Fig. 4, the transaction contracts between Gen. 1 and Gen. 2, Load 3, Load 4, and Load 5 are shown in Table 1. Gen. 1 is scheduled to generate $30 \mathrm{MW}$ to provide $20 \mathrm{MW}$ and $10 \mathrm{MW}$ for Loads 4 and 5, respectively. Gen. 2 is also scheduled to generate $80 \mathrm{MW}$ to provide $60 \mathrm{MW}$ and $20 \mathrm{MW}$ for Loads 3 and 5, respectively.

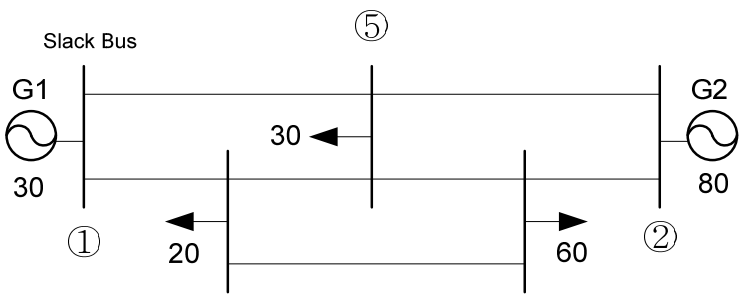

(4)

(3)

Fig. 4. Single-line diagram for the 5-bus system.

Table 1. Transaction strategy for the 5-bus system

$[\mathrm{MW}]$

\begin{tabular}{c|c|c|c|c}
\hline Bus no. & 3 & 4 & 5 & Sum \\
\hline 1 & 0 & 20 & 10 & 30 \\
\hline 2 & 60 & 0 & 20 & 80 \\
\hline Sum & 60 & 20 & 30 & 110 \\
\hline
\end{tabular}

Table 1 provides all the information about power transaction that deals only with fixed transactions. To consider flexible transactions, this paper uses a transaction ratio matrix. Note that these $\alpha_{L k}^{(i)}$ s are the same as those were in (19). All the load distribution factors can be determined in the same manner.

Based on the above results, the transaction ratio matrix $\widetilde{\mathbf{T}}_{\mathbf{G}}$ for the generator is given as follows:

$$
\widetilde{\mathbf{T}}_{\mathbf{G}}=\begin{gathered}
L_{3} \\
L_{4} \\
L_{5}
\end{gathered}\left[\begin{array}{cc}
G_{1} & G_{2} \\
0 & 0.75 \\
0.67 & 0 \\
0.33 & 0.25
\end{array}\right]
$$

where $T_{\mathrm{G} i j}$ is given by $\alpha_{L i}{ }^{(j)}$.

The above relation between the load and the generation can be expressed as follows:

$$
\begin{array}{r}
\mathbf{P}_{\mathbf{G}}=\mathbf{G D} \cdot \mathbf{1}_{n}=\widetilde{\mathbf{T}}_{\mathbf{L}} \mathbf{P}_{\mathbf{L}} \\
\mathbf{P}_{\mathbf{L}}=\mathbf{G D}^{T} \mathbf{1}_{m}=\widetilde{\mathbf{T}}_{\mathbf{G}} \mathbf{P}_{\mathbf{G}}
\end{array}
$$

where GD is the well-known transaction matrix [28], $\mathbf{1}_{p}$ is a $(p \times 1)$ vector in which all the elements are filled with one, and $\mathbf{P}_{\mathbf{L}}$ and $\mathbf{P}_{\mathbf{G}}$ are the $(m \times 1)$ load vector and $(n \times 1)$ generation vector, respectively. The load distribution factors are expressed as $\boldsymbol{\alpha}_{\mathbf{L}}^{(1)}=\left[\begin{array}{lllll}0 & 0 & 0 & 0.67 & 0.33\end{array}\right]^{T}$ and $\boldsymbol{\alpha}_{\mathbf{L}}^{(2)}=\left[\begin{array}{lllll}0 & 0 & 0.75 & 0 & 0.25\end{array}\right]^{T}$. Loss sensitivities can be 
calculated by substituting the load distribution factors into (19).

The load distribution factor can be expressed in the form of $\Delta P_{L i} / \Delta P_{G i}$. Here, the loss sensitivity can be more reasonably calculated, as the power transactions determine the power sink due to the incremental generation increase.

Once the bus-wise transaction power for each generation is determined, the transaction ratio matrix is constructed, and the bus-wise loss sensitivity factors can be easily calculated using (19) and (22).

When all the generation of the $i$-th bus is transacted to its load, the following equation is satisfied:

$$
\boldsymbol{\alpha}_{\mathbf{L}}^{(i)}=\mathbf{u}_{i}
$$

Therefore, the loss sensitivity factor of the $i$-th bus becomes zero, reflecting the situation of real systems better than any of the conventional methods. When a bilateral transaction occurs between two close regions, the loss sensitivity factor is small.

\subsection{Virtual Transaction Strategy: Bilateral and Pool Markets}

As the bilateral contracts involve transaction amounts between suppliers and consumers, the transaction strategy can be constructed using real transaction amounts represented in the previous section. The charging of losses from the power transactions in bilateral markets is fair.

However, when the market pool has incomplete bilateral transaction amounts, no full transaction strategy is obtained. Assuming that the transaction amount is contracted in proportion to each load in the market pool, the transaction of a load is expressed as follows:

$$
\alpha_{L i}=\frac{P_{L i}}{\sum P_{G k}}
$$

The alternative is the use of constructing the full transaction strategy. It is a virtual transaction strategy that is easily constructed by allowing free negotiations between market participants for the sake of the loss allocation only, regardless of the actual transactions. Adopting virtual transactions is highly recommended because market participants can take advantage of their own efforts in this system.

For incomplete bilateral markets, we can obtain a full transaction by allowing virtual transaction contracts between market participants for the sake of the loss allocation only (regardless of the actual transactions). None of the previous studies suggest any appropriate method to solve this case.

Even in the case where the full contracts of bilateral transactions are found, utilizing the virtual transaction method is highly recommended because market participants can take advantage of the virtual contracts through their own efforts. This allows us to obtain desirable transactions where generations and loads can be automatically canceled in the same bus. (Finding the real-flow transactions is impossible, but the virtual transactions are the closest to it among all transactions we can make within the market rules.) The virtual transaction strategy is a one-step advanced attempt to solve the relevant issue in this area. It may not be perfect, but it can be considered one of the most reasonable practical solutions to cover up incomplete information in transactions.

In the market pool, this paper proposes the proportional distribution method instead of the transaction strategy. Combining these two in an incomplete bilateral market is possible, i.e., we can apply the proportional distribution to market participants having no transactions. An example is the WSCC 9-bus system in Fig. 4. Incomplete bilateral transactions are given as G1-L5/67 MW, G2-L5/30 MW, and G3-L6/50 MW. First, a bilateral transaction strategy is constructed using the given transactions. Afterwards, the pool transaction strategy is conducted by proportionally distributing the rest of the power. The bilateral and pool transaction strategies are given in Tables 2 and 3, respectively. Finally, the total transaction strategy can be constructed by combining the two above-mentioned strategies as given in Table 4.

Table 2. Bilateral transaction strategy

$[\mathrm{MW}]$

\begin{tabular}{c|c|c|c|c}
\hline Bus no. & 1 & 2 & 3 & Sum \\
\hline 4 & 0 & 0 & 0 & 0 \\
\hline 5 & 67 & 30 & 0 & 97 \\
\hline 6 & 0 & 0 & 50 & 50 \\
\hline 7 & 0 & 0 & 0 & 0 \\
\hline 8 & 0 & 0 & 0 & 0 \\
\hline 9 & 0 & 0 & 0 & 0 \\
\hline Sum & 67 & 30 & 50 & 147 \\
\hline
\end{tabular}

Table 3. Pool transaction strategy

[MW]

\begin{tabular}{c|c|c|c|c}
\hline Bus no. & 1 & 2 & 3 & Sum \\
\hline 4 & 0 & 0 & 0 & 0 \\
\hline 5 & 0 & 22.167 & 5.833 & 28 \\
\hline 6 & 0 & 31.667 & 8.333 & 40 \\
\hline 7 & 0 & 0 & 0 & 0 \\
\hline 8 & 0 & 79.167 & 20.833 & 100 \\
\hline 9 & 0 & 0 & 0 & 0 \\
\hline Sum & 0 & 133 & 35 & 168 \\
\hline
\end{tabular}

Table 4. Total transaction strategy

\begin{tabular}{c|c|c|c|c}
\multicolumn{7}{c}{} & {$[\mathrm{MW}]$} \\
\hline Bus no. & 1 & 2 & 3 & Sum \\
\hline 4 & 0 & 0 & 0 & 0 \\
\hline 5 & 67 & 52.167 & 5.833 & 125 \\
\hline 6 & 0 & 31.667 & 58.333 & 90 \\
\hline 7 & 0 & 0 & 0 & 0 \\
\hline 8 & 0 & 79.167 & 20.833 & 100 \\
\hline 9 & 0 & 0 & 0 & 0 \\
\hline Sum & 67 & 163 & 85 & 315 \\
\hline
\end{tabular}




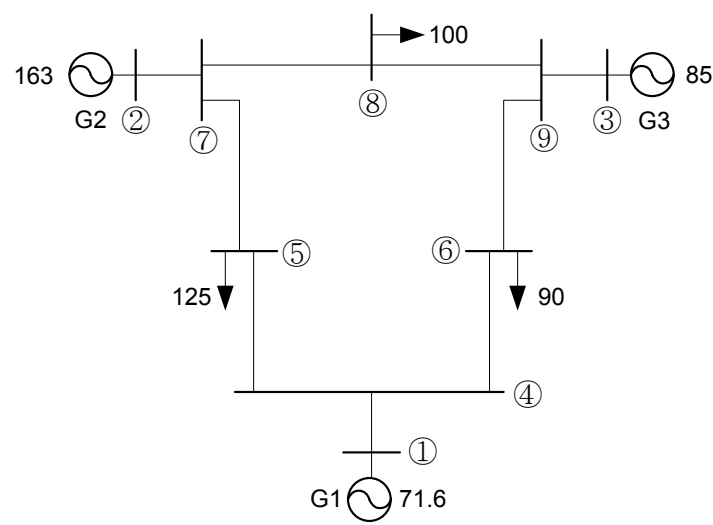

Fig. 5. Single-line diagram for the WSCC 9-bus system.

\subsection{Comparison with Galiana's Method}

The proposed method is quite similar to Galiana's method [20]. If all reactive power is neglected, the loss sensitivity $K_{p i}$ becomes the same as that given in Galiana's paper. Eq. (18) in [20] can be rewritten as $d L_{i j / s}=K_{p i} d G D_{i j}$, where $k$ is taken to be the slack bus $s$.) However, there are many differences as stated as follows:

1) Galiana considered the time integral instead of the integral path. However, the contract trajectory, altered by the acceptance order of the transactions, is arguable without providing any proper method to determine integral paths.

2) All reactive power is neglected in [20], causing some errors in loss calculation.

3) The proposed method fully considers the effects of reactive power including the zero-loading loss, which guarantees accurate loss allocations with negligible error between the actual system loss and the sum of the allocated losses.

4) This paper provides discussions on the determination of integral path while observing practical load changes. Smooth property of the marginal loss sensitivity, as observed in Fig. 2, allows us to save much computation time in calculating the average loss sensitivity, whereas Galiana's method does not. This is an important discovery for field applications.

5) A new concept of virtual transaction is introduced in this paper to achieve more reasonable loss allocations than the actual bilateral contracts.

6) The proposed method adopts a transaction ratio matrix that provides information on the ratio of the generation (the load) supplying for (receiving from) each load (generator) instead of a transaction matrix. Previously, settled transactions cannot be applied to the transaction variation in practical systems because loss is allocated after the transaction. The use of a ratio can provide more flexibility when considering the transaction variations.

7) Galiana's method uses a discrete integral, although it is expressed in the form of a time integral. Thus, his method should decrease the integration step to increase the accuracy and take drastically more computational

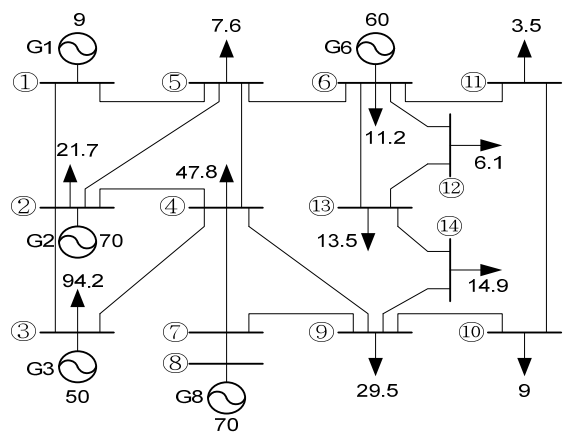

Fig. 6. Single-line diagram for the IEEE 14-bus system.

burden. However, the proposed method can provide accurate results with less computational burden (although only three points are integrated, its accuracy is not fully alleviated).

\section{Case Studies}

The WSCC 9-bus, IEEE 14-bus, England 39-bus, and IEEE 118-bus systems are tested to evaluate the proposed method. The total loss in power systems has been verified to be independent of the path the power takes through the transaction strategy. Several transaction strategies are constructed for all of the systems evaluated. Results show that the loss sensitivity factors and the allocated losses vary significantly with the transaction strategy. However, that the sum of the bus-wise allocated losses is nearly constant and is equal to the total loss is confirmed as expected.

\subsection{WSCC 9-bus System}

Four transaction strategies are used to test the WSCC 9bus system shown in Fig. 5. The transaction strategies, Cases 1-3, are given in Table 10 of the Appendix. The transaction strategy for a pool contract is evaluated in Case 4. Table 5 shows the allocated losses of the WSCC 9-bus system.

Table 5. Results of allocated losses for the WSCC-9 bus system

[MW]

\begin{tabular}{|c|c|c|c|c|c|c|c|c|}
\hline \multirow{2}{*}{ Gen } & \multicolumn{4}{|c|}{ Use of average loss sensitivity } & \multicolumn{4}{|c|}{ Use of marginal loss sensitivity } \\
\hline & Case 1 & Case 2 & Case 3 & Case 4 & Case 1 & Case 2 & Case 3 & Case 4 \\
\hline 1 & $\begin{array}{c}0.294 \\
(0.252)\end{array}$ & $\begin{array}{c}0.308 \\
(0.266)\end{array}$ & $\begin{array}{c}0.273 \\
(0.230)\end{array}$ & $\begin{array}{c}-0.113 \\
(-0.155)\end{array}$ & 0.780 & 0.814 & 0.724 & -0.122 \\
\hline 2 & $\begin{array}{c}2.890 \\
(2.788)\end{array}$ & $\begin{array}{c}2.432 \\
(2.329)\end{array}$ & $\begin{array}{c}3.635 \\
(3.533)\end{array}$ & $\begin{array}{c}3.342 \\
(3.240)\end{array}$ & 6.154 & 5.143 & 7.800 & 7.135 \\
\hline 3 & $\begin{array}{c}1.457 \\
(1.404)\end{array}$ & $\begin{array}{c}1.902 \\
(1.849)\end{array}$ & $\begin{array}{c}0.734 \\
(0.680)\end{array}$ & $\begin{array}{c}1.413 \\
(1.359)\end{array}$ & 3.108 & 4.085 & 1.518 & 3.012 \\
\hline Total & $\begin{array}{c}4.641 \\
(4.444)\end{array}$ & $\begin{array}{c}4.641 \\
(4.444)\end{array}$ & $\begin{array}{c}4.641 \\
(4.444) \\
\end{array}$ & $\begin{array}{c}4.641 \\
(4.444)\end{array}$ & 10.042 & 10.042 & 10.042 & 10.025 \\
\hline
\end{tabular}

(The figure in the parenthesis indicates the loss prior to zero-loading compensation.) 
The loss sensitivity factors vary according to the transaction strategies, i.e., the charged losses with generator buses vary and are dependent on the path. The sum of the charged losses is nearly constant and is equal to the actual loss experienced by the AC power flow after the zeroloading compensation.

\subsection{Comparisons of Path-integral and Discrete Inte- gral Methods}

The allocated losses and the actual loss are calculated for the 5-bus systems in [20] for the comparison between the proposed and Galiana's methods. Table 6 represents an acceptance order, transactions, loss sensitivity factors, and the allocated losses by discrete integral for the WSCC 9bus system. The results of the WSCC 9-bus and Galiana's 5-bus systems [20] are shown in Tables 7 and 8, respectively. The proposed method provides almost accurate results. However, in the Galiana's method, the sum of the

Table 6. Acceptance order, transactions, loss factors, and allocated losses by discrete integral for the WSCC 9-bus system

\begin{tabular}{c|c|c|c|c}
\hline $\begin{array}{c}\text { Acceptance } \\
\text { order }\end{array}$ & Transaction & MW & $\begin{array}{c}\text { Loss } \\
\text { factor }\end{array}$ & $\begin{array}{c}\text { Allocated } \\
\text { loss }\end{array}$ \\
\hline $\mathrm{T}_{1}$ & $G D_{15}$ & 62 & 0.0105 & 0.651 \\
\hline $\mathrm{T}_{2}$ & $G D_{25}$ & 63 & 0.0320 & 2.013 \\
\hline $\mathrm{T}_{3}$ & $G D_{16}$ & 5 & -0.00233 & -0.012 \\
\hline $\mathrm{T}_{4}$ & $G D_{36}$ & 85 & 0.0519 & 4.407 \\
\hline $\mathrm{T}_{5}$ & $G D_{28}$ & 100 & 0.0133 & 1.329 \\
\hline
\end{tabular}

Table 7. Comparison of the allocated losses and the their sums for the WSCC 9-bus system (case 2)

\begin{tabular}{c|c|c}
\hline \multirow{2}{*}{ Gen } & \multicolumn{2}{|c}{ Allocated losses [MW] } \\
\cline { 2 - 3 } & Discrete integral & Proposed \\
\hline 1 & 0.639 & 0.308 \\
\hline 2 & 3.342 & 2.432 \\
\hline 3 & 4.407 & 1.902 \\
\hline Loss sum & 8.388 & 4.641 \\
\hline Difference & 3.747 & 0.000 \\
\hline Actual loss & \multicolumn{2}{|c}{4.641} \\
\hline
\end{tabular}

Table 8. Comparison of the allocated losses and their sums for the Galiana 5-bus system

\begin{tabular}{c|c|c}
\hline \multirow{2}{*}{ Gen } & \multicolumn{2}{|c}{ Allocated losses [MW] } \\
\cline { 2 - 3 } & Discrete integral & Proposed \\
\hline 1 & 51.8 & 38.193 \\
\hline 2 & 7.0 & 4.818 \\
\hline 3 & -49.3 & -48.314 \\
\hline 4 & 62.1 & 51.501 \\
\hline 5 & 0.2 & 4.028 \\
\hline Loss sum & 71.8 & 50.225 \\
\hline Difference & 15.88 & 0.099 \\
\hline Actual loss & \multicolumn{2}{|c}{50.132} \\
\hline
\end{tabular}

allocated losses is different from the actual loss. It does not guarantee high accuracy because it accepts the transactions in some orders. If the computation time allows, we can suppose that all the transactions be divided into 10 (or 100) steps. Each step accepts only $10 \%$ (or $1 \%$ ) of the total transactions. We will then have 10 (or 100) times of transactions through 10 (or 100) times bidding. Although computation burden is increased drastically, Galiana's method produces more accurate loss allocations.

\subsection{IEEE 14-bus System}

The proposed method is also applied to the IEEE 14-bus system shown in Fig. 6. The transaction strategies from Cases 1-3 are given in Table 11 of the Appendix.

The transaction strategy for a pool contract is evaluated in Case 4. Table 9 shows the results of the loss allocation of the IEEE 14-bus system. The results indicate that the marginal/average loss sensitivities vary with the transaction strategy as the latter determines the integral path. Conventional approaches have been somewhat unfair in such a way that the sum of the allocated losses doubles the actual loss. Here, note that the proposed method improves the reliability of the loss allocation by showing that the sum of the allocated losses is almost equal to the actual loss.

Table 9. Results of allocated loss for the IEEE 14-bus system

\begin{tabular}{c|c|c|c|c|c|c|c|c}
\hline \multirow{2}{*}{ Gen } & \multicolumn{7}{|c|}{ Use of average loss sensitivity } & \multicolumn{3}{c}{ Use of marginal loss sensitivity } \\
\cline { 2 - 9 } & Case 1 & Case 2 & Case 3 & Case 4 & Case 1 & Case 2 & Case 3 & Case 4 \\
\hline \multirow{2}{*}{1} & $\begin{array}{c}0.018 \\
(-0.000)\end{array}$ & $\begin{array}{c}-0.003 \\
(-0.021)\end{array}$ & $\begin{array}{c}0.017 \\
(-0.001)\end{array}$ & $\begin{array}{c}0.078 \\
(0.060)\end{array}$ & 0.026 & -0.017 & 0.025 & 0.172 \\
\hline 2 & $\begin{array}{c}0.570 \\
(0.431)\end{array}$ & $\begin{array}{c}0.606 \\
(0.467)\end{array}$ & $\begin{array}{c}0.470 \\
(0.331)\end{array}$ & $\begin{array}{c}0.768 \\
(0.629)\end{array}$ & 1.042 & 1.118 & 0.718 & 1.466 \\
\hline \multirow{2}{*}{3} & $\begin{array}{c}0.099 \\
(0.000)\end{array}$ & $\begin{array}{c}0.099 \\
(0.000)\end{array}$ & $\begin{array}{c}0.099 \\
(0.000)\end{array}$ & $\begin{array}{c}0.024 \\
(-0.076)\end{array}$ & 0.000 & 0.000 & 0.000 & -0.304 \\
\hline \multirow{2}{*}{6} & $\begin{array}{c}0.673 \\
(0.554)\end{array}$ & $\begin{array}{c}0.698 \\
(0.578)\end{array}$ & $\begin{array}{c}0.803 \\
(0.683)\end{array}$ & $\begin{array}{c}0.611 \\
(0.492)\end{array}$ & 1.162 & 1.217 & 1.440 & 1.141 \\
\hline 8 & $\begin{array}{c}0.373 \\
(0.234)\end{array}$ & $\begin{array}{c}0.333 \\
(0.194)\end{array}$ & $\begin{array}{c}0.344 \\
(0.205)\end{array}$ & $\begin{array}{c}0.256 \\
(0.113)\end{array}$ & 0.655 & 0.567 & 0.702 & 0.410 \\
\hline \multirow{2}{*}{ Total } & $\begin{array}{c}1.734 \\
(1.218)\end{array}$ & $\begin{array}{c}1.734 \\
(1.218)\end{array}$ & $\begin{array}{c}1.734 \\
(1.218)\end{array}$ & $\begin{array}{c}1.734 \\
(1.218)\end{array}$ & 2.885 & 2.885 & 2.885 & 2.885 \\
\hline \multicolumn{7}{c}{ Actual loss: 1.734 (zero-loading loss: 0.515) } \\
\hline
\end{tabular}

(The figure in parenthesis indicates the loss prior to zero-loading compensation)

\subsection{New England 39-bus System and IEEE 118-bus System}

To apply the proposed method to larger systems, the algorithm is tested using the New England 39-bus and IEEE 118-bus systems. The reactive power limit in the bus is not considered to simplify the test. As shown above, it is confirmed that the sum of the allocated losses is almost equal to the actual loss, and the bus-wise losses vary with the transaction strategy.

In the case of the pool contract, the actual losses are 132.812 MW and 42.733 MW, with the differences be- 
tween the calculated and actual loss being $2.84 \times 10^{-5} \mathrm{MW}$ and $0.020 \mathrm{MW}$ for the New England 39-bus system and the IEEE 118-bus system, respectively. Similar differences are observed in the case of the bilateral contract with the transaction strategy: $6.65 \times 10^{-5} \mathrm{MW}$ and $0.054 \mathrm{MW}$ for the New England 39-bus system and the IEEE 118-bus system, respectively. The differences in all cases are small enough to be neglected or proportionally distributed to each generator. The numerical results show that the proposed algorithm can be applied well to real systems.

Table 10 shows the results of the sample tests with several integral step sizes. When the step size is very large $($ step $=0.5)$, the proposed method produces very accurate results (error is within $0.1 \%$ ). This fact is very important for reducing the computational burden for practical field application to large power systems.

Table 10. Actual and sum of the allocated losses and error in the New England 39-bus and IEEE 118-bus systems

\begin{tabular}{c|c|c|c|c}
\hline \multicolumn{2}{c|}{ Step size } & 0.1 & 0.2 & 0.5 \\
\hline \multirow{2}{*}{$\begin{array}{c}\text { New England } \\
\text { 39-bus system }\end{array}$} & Simpson & 42.7329 & 42.7442 & 42.7675 \\
\cline { 2 - 5 } & Error (\%) & $7.02 \mathrm{E}-05$ & 0.026 & 0.081 \\
\cline { 2 - 5 } & Trapezoidal & 42.7411 & 42.8330 & 43.4681 \\
\cline { 2 - 5 } Actual:42.7329 & Error (\%) & 0.019 & 0.23 & 1.72 \\
\hline \multirow{2}{*}{$\begin{array}{c}\text { IEEE 118-bus } \\
\text { system }\end{array}$} & Simpson & 132.8316 & 132.8525 & 132.8516 \\
\cline { 2 - 5 } & Error (\%) & 0.015 & 0.031 & 0.030 \\
\cline { 2 - 5 } Actual:132.8116 & Trapezoidal & 132.8791 & 133.0216 & 134.0148 \\
\cline { 2 - 5 } & Error (\%) & 0.051 & 0.16 & 0.91 \\
\hline
\end{tabular}

\section{Conclusions}

This paper proposes a new transmission loss allocation method that can provide a reliable way to satisfy the path independency of the total sum of allocated losses. We also compare this method with Galiana's discrete integral method. Bus-wise allocated losses vary according to the transaction strategy. However, the sum of the allocated losses of each bus, including zero-loading loss, is nearly constant and equal to the actual loss. The proposed method solves the problem of conventional approaches that use the sensitivity method. These approaches result in the total sum of allocated losses becoming almost twice of the actual loss. Slack-bus-independent loss sensitivity is adopted to minimize the variation in the sensitivity that occurs when a new slack bus is selected. This paper introduces a loss allocation method for pool and bilateral markets. The proposed method is applied to the WSCC 9-bus, IEEE 14bus, New England 39-bus, and IEEE 118-bus systems. The results indicate that transmission loss can be allocated fairly and accurately while still considering the transaction strategy.

\section{Appendix A}

Path dependency for partial integral

To help understand path dependency by path integral (line integral), illustrative examples are presented as follows.

$$
f(x, y, z)=x^{2}+x y+y^{2}+z^{2}+2 y z
$$

Eq. (A.1) is reconstructed in the same manner as (10), which is given as follows:

$$
\begin{aligned}
P & =f\left(x_{1}, y_{1}, z_{1}\right) \\
& ={ }_{c} \int_{(0,0,0)}^{\left(x_{1}, y_{1}, z_{1}\right)}[(2 x+y) d x+(x+2 y+2 z) d y+(2 z+2 y) d z]
\end{aligned}
$$

It is assured that the above integration form, (A.2), satisfies the condition of (11), and total integration $\mathrm{P}$ becomes path independent. However, each partial term is path dependent. For example, one point on the integral path, $(x, y, z)$, is given as $x=x_{1} \lambda, y=y_{1} \lambda$, and $z=z_{1} \lambda$ if from $(0,0,0)$ to $\left(x_{1}, y_{1}, z_{1}\right)$ a linear integral path $\bar{c}$ is selected, where $\lambda \in[0,1]$. Thus, each of the partial integration and total integration are manipulated as follows:

$$
\begin{aligned}
P^{(1)} & ={ }_{\bar{c}} \int_{(0,0,0)}^{\left(x_{1}, y_{1}, z_{1}\right)}(2 x+y) d x=\int_{0}^{1}\left(2 x_{1} \lambda+y_{1} \lambda\right) x_{1} d \lambda \\
& =\left.\left(2 x_{1}^{2}+y_{1} x_{1}\right) \cdot \frac{1}{2} \lambda^{2}\right|_{0} ^{1}=x_{1}^{2}+\frac{1}{2} x_{1} y_{1} \\
P^{(2)}= & { }_{\bar{c}} \int_{(0,0,0)}^{\left(x_{1}, y_{1}, z_{1}\right)}(x+2 y+2 z) d y \\
= & \int_{0}^{1}\left(x_{1} \lambda+2 y_{1} \lambda+2 z_{1} \lambda\right) y_{1} d \lambda=\frac{1}{2} x_{1} y_{1}+y_{1}^{2}+y_{1} z_{1} \\
P^{(3)}= & \int_{\bar{c}}^{\left(x_{1}, y_{1}, z_{1}\right)}(2 z+2 y) d z=\int_{0}^{1}\left(2 z_{1} \lambda+2 y_{1} \lambda\right) z_{1} d \lambda \\
= & z_{1}^{2}+y_{1} z_{1} \\
P=P^{(1)}+P^{(2)}+P^{(3)} & =x_{1}^{2}+y_{1}^{2}+z_{1}^{2}+x_{1} y_{1}+2 y_{1} z_{1}
\end{aligned}
$$

In turn, if path $\bar{c}_{1}$ is constructed by combining paths $\bar{c}_{a}$ and $\bar{c}_{b}$ which are linear between $(0,0,0)$ and $\left(x_{1}, y_{1}, 0\right)$, and between $\left(x_{1}, y_{1}, 0\right)$ and $\left(x_{1}, y_{1}, z_{1}\right)$ respectively, each of the partial integration is given as: 


$$
\begin{aligned}
P^{(1)}= & \bar{c}_{1} \int_{(0,0,0)}^{\left(x_{1}, y_{1}, z_{1}\right)}(2 x+y) d x \\
= & \bar{c}_{a} \int_{(0,0,0)}^{\left(x_{1}, y_{1}, 0\right)}(2 x+y) d x+\bar{c}_{\bar{c}_{b}} \int_{\left(x_{1}, y_{1}, 0\right)}^{\left(x_{1}, y_{1}, z_{1}\right)}(2 x+y) d x \quad \text { (A.7) } \\
= & \int_{0}^{1}\left(2 x_{1} \lambda+y_{1} \lambda\right) x_{1} d \lambda+0=x_{1}^{2}+\frac{1}{2} x_{1} y_{1} \\
P^{(2)}={ }_{\bar{c}_{1}} \int_{(0,0,0)}^{\left(x_{1}, y_{1}, z_{1}\right)}(x+2 y+2 z) d y & (\mathrm{~A} .8) \\
= & { }_{\bar{c}_{a}} \int_{(0,0,0)}^{\left(x_{1}, y_{1}, 0\right)}(x+2 y+2 z) d y+\bar{c}_{\bar{c}_{b}} \int_{\left(x_{1}, y_{1}, 0\right)}^{\left(x_{1}, y_{1}, z_{1}\right)}(x+2 y+2 z) d y \\
= & \frac{1}{2} x_{1} y_{1}+y_{1}^{2} \\
P^{(3)} & =\bar{c}_{\bar{c}_{1}} \int_{(0,0,0)}^{\left(x_{1}, y_{1}, z_{1}\right)}(2 z+2 y) d z \\
& ={ }_{\bar{c}_{a}} \int_{(0,0,0)}^{\left(x_{1}, y_{1}, 0\right)}(2 z+2 y) d z+\bar{c}_{\bar{c}_{b}} \int_{\left(x_{1}, y_{1}, 0\right)}^{\left(x_{1}, y_{1}, z_{1}\right)}(2 z+2 y) d z \\
& =z_{1}^{2}+2 y_{1} z_{1} \\
P=x_{1}^{2}+y_{1}^{2}+z_{1}^{2}+x_{1} y_{1}+2 y_{1} z_{1} & (\mathrm{~A} .10)
\end{aligned}
$$

In the above manipulation, the integrations on paths $\bar{c}_{a}$ and $\bar{c}_{b}$ use path parameter $\lambda$. Each of the partial integration term varies with the path, which means that the partial term $P^{(i)}$ is path dependent. Each of the partial integration term is always path dependent if a function is reconstructed by the sum of the partial integration terms. However, note that without reference to the path, the sum of each partial integration term is always the same as (A.1), that is, path independent.

\section{Appendix B}

Table 11. Transaction strategy for the WSCC 9-bus system

\begin{tabular}{c|c|c|c}
\hline & Case 1 & Case 2 & Case 3 \\
\hline G1 & $38.4 / \mathrm{L} 5,28.6 / \mathrm{L} 6$ & $62 / \mathrm{L} 5,5 / \mathrm{L} 6$ & $67 / \mathrm{L} 6$ \\
\hline G2 & $86.6 / \mathrm{L} 5,76.4 / \mathrm{L} 8$ & $63 / \mathrm{L} 5,100 / \mathrm{L} 8$ & $125 / \mathrm{L} 5,38 / \mathrm{L} 8$ \\
\hline G3 & $61.4 / \mathrm{L} 6,23.6 / \mathrm{L} 8$ & $85 / \mathrm{L} 6$ & $23 / \mathrm{L} 6,62 / \mathrm{L} 8$ \\
\hline
\end{tabular}

Table 12. Transaction strategy for the IEEE 14-bus system

\begin{tabular}{c|c|c|c}
\hline & Case 1 & Case 2 & Case 3 \\
\hline G1 & $3.8 / \mathrm{L} 2,5.2 / \mathrm{L} 5$ & $9 / \mathrm{L} 2$ & $4 / \mathrm{L} 2,5 / \mathrm{L} 5$ \\
\hline \multirow{2}{*}{ G2 } & $17.9 / \mathrm{L} 2,27.3 / \mathrm{L} 3$, & $12.7 / \mathrm{L} 2,27.3 / \mathrm{L} 3$, & $17.7 / \mathrm{L} 2,4 / \mathrm{L} 3$, \\
& $22.4 / \mathrm{L} 4,2.4 / \mathrm{L} 5$ & $30 / \mathrm{L} 4$ & $47.8 / \mathrm{L} 4,0.5 / \mathrm{L} 5$ \\
\hline G3 & $50 / \mathrm{L} 3$ & $50 / \mathrm{L} 3$ & $50 / \mathrm{L} 3$ \\
\hline \multirow{4}{*}{ G6 } & $11.8 / \mathrm{L} 4,11.2 / \mathrm{L} 6$, & $7.6 / \mathrm{L} 5,11.2 / \mathrm{L} 6$, & $2.1 / \mathrm{L} 5,11.2 / \mathrm{L} 6$, \\
& $6 / \mathrm{L} 10,3.5 / \mathrm{L} 11$, & $9 / \mathrm{L} 10,3.5 / \mathrm{L} 11$, & $8.7 / \mathrm{L} 10,3.5 / \mathrm{L} 11$, \\
& $6.1 / \mathrm{L} 12,13.5 / \mathrm{L} 13$, & $6.1 / \mathrm{L} 12,13.5 / \mathrm{L} 13$, & $6.1 / \mathrm{L} 12,13.5 / \mathrm{L} 13$, \\
& $7.9 / \mathrm{L} 14$ & $9.1 / \mathrm{L} 14$ & $14.9 / \mathrm{L} 14$ \\
\hline \multirow{3}{*}{ G8 } & $16.9 / \mathrm{L} 3,13.6 / \mathrm{L} 4$, & $16.9 / \mathrm{L} 3,17.8 / \mathrm{L} 4$, & $40.2 / \mathrm{L} 3,29.5 / \mathrm{L} 9$, \\
& $29.5 / \mathrm{L} 9,3 / \mathrm{L} 10$, & $29.5 / \mathrm{L} 9,5.8 / \mathrm{L} 14$ & $0.3 / \mathrm{L} 10$ \\
& $7 / \mathrm{L} 14$ & &
\end{tabular}

Tables 10 and 11 show the transaction strategies of cases 1-3 for the WSCC 9-bus system and the IEEE 14-bus system, respectively (legend: 10/L1 means $10 \mathrm{MW}$ to load at bus 1).

\section{References}

[1] E. Litvinov, T. Zheng, G Rosenwald and P. Shamsollahi, "Marginal loss modeling in LMP Calculation," IEEE Trans. Power Syst., Vol. 19, No. 2, May 2004.

[2] J. B. Cardell, "Marginal loss pricing for hours with transmission congestion," IEEE Trans. Power Syst., Vol. 22, No. 4, Nov. 2007.

[3] F. Li and R. Bo, "DCOPF-based LMP simulation: algorithm, comparison with ACOPF, and sensitivity," IEEE Trans. Power Syst., Vol. 22, No. 4, Nov. 2007.

[4] G. Gross and S. Tao, "A Physical-Flow-Based Approach to Allocating Transmission Losses in a Transaction Framework," IEEE Trans. Power Syst., Vol. 15, No. 2, May 2000.

[5] R. Nadira et al., "Bulk Transmission System Loss Analysis," IEEE Trans. Power Syst., vol. 8, no. 2, pp. 405-417, May 1993.

[6] Q. Ding and A. Abur, "Transmission loss Allocation in a Multiple-Transaction Framework," IEEE Trans. Power Syst., Vol. 19, No. 1, Feb. 2004.

[7] A. J. Conejo, F. D. Galiana, and I. Kockar, "Z-bus Loss Allocation," IEEE Trans. Power Syst., Vol. 16, No. 1, Feb. 2001

[8] J. J. Grainger and W.D. Stevenson, Jr., Power System Analysis, McGraw-Hill, Inc. pp. 329-356 1994.

[9] A. J. Conejo, J. M. Arroyo, N. Alguacil, and A. L. Guijarro, "Transmission Loss Allocation: A Comparison of Different Practical Algorithms," IEEE Trans. Power Syst., Vol. 17, No. 3, Aug. 2002.

[10] A. G. Exposito, J. M. R. Santos, T. G. Garcia, and E.A. R. Velasco, "Fair Allocation of Transmission Power Losses," IEEE Trans. Power Syst., Vol. 15, No. 1, Feb. 2000.

[11] S. M. Abdelkader, "Allocating transmission loss to loads and generators through complex power flow tracing," IET Gener. Transm. Distrib., Vol. 1, No. 4, July 2007.

[12] Y. P. Molina, R. B. Prada, and O. S. Mendez, "On the Partition of Transmission Losses Among Generators," IEEE Trans. Power Syst., Vol. 23, No. 4, Nov. 2008.

[13] H. X. Wang, R. Liu and W. D. Li, "Transmission Loss Allocation Based on Circuit Theories and Orthogonal Projection," IEEE Trans. Power Syst., Vol. 24, No. 2, May 2009.

[14] D. Songhuai, Z. Xinghua, M. Lu, and X. Hui, "A novel nucleolus-based loss allocation method in bilateral electricity markets," IEEE Trans. Power Syst., Vol. 21, No. 1, Feb. 2006.

[15] J. N. Y. Cheung, T. Czaszejko and A. B. Morton, "Transmission loss evaluation in an open electricity 
market using an incremental method," IET Gener. Transm. Distrib., Vol. 1, No. 1, Jan. 2007.

[16] K. I. Min, S. H. Ha, S. W. Lee, and Y. H. Moon, "Transmission Loss Allocation Algorithm Using Path-Integral Based on Transaction Strategy," IEEE Trans. Power Syst., Vol. 25, No. 1, Feb. 2010.

[17] C. S. You, K. I. Min, J. G. Lee, and Y. H. Moon, “Allocation of transmission loss for determination of locational spot pricing," KIEE J. Electr. Eng. Technol., Vol. 2, No. 2, pp. 194-200, 2007.

[18] Q. Ding and A. Abur, "A unified transmission loss allocation method," Int. J. Elect. Power Energy Syst., Vol. 29, No. 5, June 2007.

[19] E. Carpaneto, G. Chicco, and J. S. Akilimali, "Loss Partitioning and Loss Allocation in Three-Phase Radial Distribution Systems With Distributed Generation," IEEE Trans. Power Syst., Vol. 23, No. 3, Aug. 2008.

[20] F. D. Galiana and M. Phelan, "Allocation of transmission losses to bilateral contracts in a competitive environment," IEEE Trans. Power Syst., Vol. 15, No. 1, pp. 143-150, Feb. 2000.

[21] F. D. Galiana, A. J. Conejo, and I. Kochar, "Incremental transmission loss allocation under pool dispatch," IEEE Trans. Power Syst., Vol. 17, No. 1, pp. 26-33, 2002

[22] Y. H. Moon, B. K. Choi, H. S. Ryu, J. S. Jung, and H. M. Park, "Slack-Bus Independent Penalty Factor for Spot Pricing under Deregulation," in Proc. IEEE PES Summer Meeting, Vol. 4, pp. 1017-1021, 2000.

[23] Y. H. Moon, H. S. Hong, H. S. Ryu, B. K. Choi, and J. D. Park, "Slack-bus independent penalty factor for regional spot pricing under deregulation," Int. J. Elect. Power and Energy Syst., Vol. 24, pp. 821-826, 2002.

[24] A. Fradi, S. Brignone and B. F. Wollenberg, "Calculation of Energy Transaction Allocation Factors," IEEE Trans. Power Syst., Vol. 16, No. 2, pp. 266-272, May 2001.
[25] A. M. Leite da Silve and J. G. de Carvalho Costa, "Transmission Loss Allocation: Part I - Single Energy Market," IEEE Trans. Power Syst., Vol. 18, No. 4, Nov. 2003.

[26] F. Gubina, D. Grgic, and I. Banic, "A Method for Determining the Generators' Share in a Consumer Load," IEEE Trans. Power Syst., Vol. 15, No. 4, Nov. 2000.

[27] D. Kirschen, R. Allan, and G. Strbac, "Contributions of Individual Generators to Loads and Flows," IEEE Trans. Power Syst., Vol. 12, No. 1, Feb.1997.

[28] James Stewart, Calculus. $6^{\text {th }}$ Ed., Thomson, 2008.

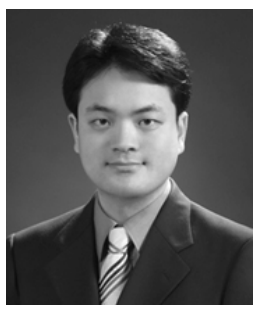

Kyung-II Min received his B.S. degree from Hongik University, Seoul, Korea, in 2001, and his M.S. and Ph.D. degrees from Yonsei University, Seoul, Korea, in 2003 and 2010, respectively. He was a researcher with the Automation Technology Research Institute, Yonsei University. He is currently with Samsung Electronics, as an engineer working on facility planning. His interests include power system operation and economics, power system stability, state estimation, and power system protection.

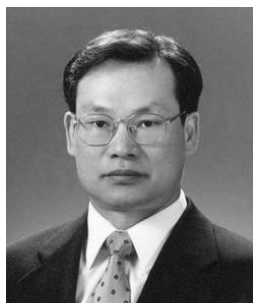

Young-Hyun Moon received his B.S. and M.S. degrees from Seoul National University, Seoul, Korea, in 1975 and 1978, respectively, and his Ph.D. from the Oregon State University in 1983. He is currently a Professor of Electrical and Electronic Engineering at Yonsei University, Seoul, Korea. His major research fields are power system operation and economics, stability analysis, and state estimation in power systems. 